\title{
A Class of Organopolysulfides as Liquid Cathode Materials for
}

\section{High Energy Density Lithium Batteries}

Amruth Bhargav ${ }^{\dagger}$, Michaela Elaine Bell ${ }^{\dagger}$, Jonathan Karty§, Yi Cui ${ }^{\dagger \#}$, and Yongzhu Fu ${ }^{\dagger \neq *}$

$\dagger$ Department of Mechanical Engineering, Indiana University-Purdue University Indianapolis, Indianapolis, IN 46202, United States

† College of Chemistry and Molecular Engineering, Zhengzhou University, Zhengzhou 450001, China

$\S$ Department of Chemistry, Indiana University, Bloomington, IN 47405, United States

\# School of Mechanical Engineering, Purdue University, West Lafayette, IN 47907, United States

*Corresponding author: yfu@zzu.edu.cn (Y. Fu)

KEYWORDS - organopolysulfide, phenyl polysulfide, lithium battery, specific energy, energy density

\begin{abstract}
Sulfur-based cathodes are promising to enable high energy density lithium-sulfur batteries; however, elemental sulfur as active material faces several challenges including undesirable volume change ( $\sim 80 \%)$ when completely reduced and high dependence on liquid electrolyte wherein an electrolyte/sulfur ratio $>10 \mu \mathrm{L} \mathrm{mg}^{-1}$ is required for high material utilization. These limit the attainable energy densities of these batteries. Herein, we introduce a new class of phenyl polysulfides $\mathrm{C}_{6} \mathrm{H}_{5} \mathrm{~S}_{\mathrm{x}} \mathrm{C}_{6} \mathrm{H}_{5}(4 \leq \mathrm{x} \leq 6)$ as liquid cathode materials synthesized in a facile and scalable route to mitigate these setbacks. These polysulfides possess sufficiently
\end{abstract}


high theoretical specific capacities, specific energies, and energy densities. Spectroscopic techniques verify their chemical composition and computation shows that the volume change when reduced is about 37\%. Lithium half-cell testing shows that phenyl hexasulfide $\left(\mathrm{C}_{6} \mathrm{H}_{5} \mathrm{~S}_{6} \mathrm{C}_{6} \mathrm{H}_{5}\right)$ can provide a specific capacity of $650 \mathrm{mAh} \mathrm{g}^{-1}$ and capacity retention of $80 \%$ through 500 cycles at 1C rate along with superlative performance up to 10C. Furthermore, 1,302 Wh $\mathrm{kg}^{-1}$ and 1,720 Wh L $\mathrm{L}^{-1}$ are achievable at a low electrolyte/active material ratio, i.e., $3 \mu \mathrm{L} \mathrm{mg}$ ${ }^{1}$. This work adds new members to the cathode family for Li-S batteries, reduces the gap between the theoretical and practical energy densities of batteries, and provides a new direction for the development of alternative high-capacity cathode materials.

From waking up to alarms on a cellphone to getting around in electric cars, our everyday lives are progressively relying on rechargeable batteries. The dramatic advances in these systems have been the major driving force for scientists to explore new materials that enable batteries with higher specific energy and energy density. Society has been so far reliant on solid metal oxide cathodes for lithium-ion (Li-ion) batteries. ${ }^{1-2}$ However, the inherently low specific capacities of these materials limit the achievable specific energy. ${ }^{3-4}$ This roadblock led to the investigations into solid conversion cathodes such as elemental sulfur which offers an order of magnitude higher specific capacity thus improving the specific energy. ${ }^{5-6}$ Despite being a promising cathode material, an intrinsic volume change ( $\sim 80 \%)$ occurring during conversion of sulfur to lithium sulfide necessitates cathode substrates that possess structural stability throughout the cycle life of the cell. ${ }^{5}$ In addition, complete conversion in ether electrolyte requires a high electrolyte/sulfur ratio, e.g., $\geq 10 \mu \mathrm{L} \mathrm{mg}^{-1}$, which significantly limits the specific energy of lithium-sulfur (Li-S) batteries. ${ }^{7-11}$ 
Research efforts aimed at accommodating this volume change have focused on designing cathode to physically flex during battery operation by employing sulfur wrapped cathodes ${ }^{12-13}$, confinement in core-shell structures ${ }^{14-15}$, flexible polymers and the like. ${ }^{16-17}$ Unfortunately, during long term cycling, these solutions result in microstructural changes in the cathode which negatively affect the performance. ${ }^{18}$ Furthermore, this effect is further exacerbated at higher sulfur loadings. ${ }^{19}$ An alternative strategy to cathode stabilization relies upon liquid sulfurcontaining compounds, where the volume change between the lithiated and delithiated materials is greatly reduced. Herein, we utilize a facile and scalable process to synthesize phenyl polysulfides namely phenyl tetrasulfide (PTS, $\mathrm{C}_{6} \mathrm{H}_{5} \mathrm{~S}_{4} \mathrm{C}_{6} \mathrm{H}_{5}, \mathrm{PhS}_{4} \mathrm{Ph}$ ), phenyl pentasulfide (PPS, $\mathrm{PhS}_{5} \mathrm{Ph}$ ), and phenyl hexasulfide (PHS, $\mathrm{PhS}_{6} \mathrm{Ph}$ ) in pure forms. In these compounds, a linear sulfur chain is bonded with two phenyl groups on the ends. Each polysulfide molecule can take several $\mathrm{Li}^{+}$and $\mathrm{e}^{-}$when reduced; therefore, possess a high specific capacity and energy. The volume change when utilizing these materials is about 37\% (see supporting for computation), which is less than half that of the sulfur cathode in Li-S batteries thus significantly reducing internal electrode stresses. This enables the cathode to accommodate the chemical transitions over a few hundred cycles while still maintaining mechanical integrity and ensuring electrical contact with the active material. In addition, this class of liquid cathode materials allows for high material utilization at low electrolyte/active material ratios (i.e., $3 \mu \mathrm{L} \mathrm{mg}^{-1}$ ) thus reducing the fraction of inactive cell components. These unique properties can be leveraged for developing lithium batteries with long cycle life and high energy density for practical applications.

In this letter, these new polysulfides were synthesized in a facile and scalable route and their chemical composition was verified using different spectroscopic techniques. Their electrochemical, material and physical transformations occurring on the cathode in lithium half- 
cells were probed to understand their reduction processes in batteries. Furthermore, performances under high rate and also low electrolyte to active material ratios were evaluated, which shows their potential to overcome the intrinsic issues of sulfur cathodes.

\section{Results and Discussion}

Bis(aryl) polysulfides such as phenyl polysulfides and benzyl polysulfides have been synthesized commonly through the reaction of the corresponding thiols with sulfur monochloride, ${ }^{20-22}$ reaction of corresponding aryl halides with alkali polysulfides, ${ }^{23-24}$ and also the base-catalyzed reaction of thiols with elemental sulfur ${ }^{25}$ among others. ${ }^{26}$ These techniques rely on a variety of solvents, catalysts and non-ambient conditions and result in a mixture of reaction products thus requiring further extraction/purification steps before obtaining the final products in pure form. Herein, we introduce a solvent-free and catalyst-free technique to synthesize phenyl polysulfides that yields the required product in a single step. The reaction involves the simple mixing and stirring of the starting thiol, i.e., benzenethiol (PhSH) with elemental sulfur in the appropriate stoichiometric ratio as visualized along with the equation in Figure 1a. The high acidity of the thiol group facilitates the ring-opening of the cyclo-octasulfur leading to the formation of phenyl polysulfanes $\left(\mathrm{PhS}_{\mathrm{x}} \mathrm{H}\right) .{ }^{25}$ The instability of these high order polysulfanes results in intermolecular condensation and elimination of $\mathrm{H}_{2} \mathrm{~S}$ gas leading to the formation of the required order of phenyl polysulfide. ${ }^{25-26}$ The presence of $\mathrm{H}_{2} \mathrm{~S}$ can be detected through the blackening on exposure of lead acetate test strips (shown in Figure 1a) and can also be observed in the bubbling of the reaction mixture (supporting Figure S1). The $\mathrm{H}_{2} \mathrm{~S}$ evolution greatly subsides in 1 hour and ceases at about 5 hours. Continuous agitation until 6 hours removes almost all the dissolved gas leaving behind the oily polysulfide liquid. Furthermore, 
upon scaling, the $\mathrm{H}_{2} \mathrm{~S}$ released can be collected from the reaction vessel and the sulfur can be recovered from it using the Claus process thus regenerating the starting material and mitigating the toxic impact of $\mathrm{H}_{2} \mathrm{~S}$.

Two equivalents of PhSH react with 3 equivalents of sulfur to yield phenyl tetrasulfide (PTS, $\mathrm{PhS}_{4} \mathrm{Ph}$ ). Similarly, 4 equivalents of sulfur give phenyl pentasulfide (PPS, $\left.\mathrm{PhS}_{5} \mathrm{Ph}\right)$ and 5 equivalents result in phenyl hexasulfide (PHS, $\mathrm{PhS}_{6} \mathrm{Ph}$ ) which are shown in Figure 1b. As expected, the density of these liquids almost linearly increases with polysulfide order. This results in the gravimetric specific capacity and volumetric capacity density increase with the order of phenyl polysulfide as summarized in Figure 1c. PHS has a specific capacity of 774.5 $\mathrm{mAh} \mathrm{g}^{-1}$ and a capacity density of 1,022.4 $\mathrm{mAh} \mathrm{mL}^{-1}$ which are, respectively, nearly 4 times and twice that of state-of-the-art cathode materials used in Li-ion batteries. ${ }^{27}$ On collating the energy increase with polysulfide order as described by Figure 1d, we see that all the three polysulfides have a higher specific energy than metal oxide cathode materials, while PPS and PHS are able to surpass them in terms of energy density. This comparison of theoretical capacity and energy values clearly establishes the great promise this class of materials hold in surpassing the current cathode materials used in Li-ion batteries.

Upon their synthesis, different spectroscopic techniques were used to corroborate the composition of the polysulfides. Firstly, Fourier Transform Infrared (FTIR) spectroscopy was conducted to confirm the conversion of thiol to polysulfide. The S-H bond stretch, $v(\mathrm{SH})$, is typically present in the $2500-2600 \mathrm{~cm}^{-1}$ range as shown in the IR spectrum in Figure $2 \mathrm{a} .{ }^{28}$ This is evident in the spectrum of PhSH whereas this peak is absent in the spectra of PTS, PPS, and PHS marking the conversion of the thiol into corresponding polysulfides. Characteristic peaks of the phenyl polysulfide bonds are visible in the $400-550 \mathrm{~cm}^{-1}$ region (supporting Figure S2). Here, 
weak peaks attributed to S-S bond stretching mode can be seen in 480-500 $\mathrm{cm}^{-1}$ band along with the stronger peak at about $470 \mathrm{~cm}^{-1}$ owing to the out-of-plane deformation of the phenyl rings due to the presence of polysulfide linkages. ${ }^{29-30}$ Next, on establishing the conversion to polysulfides, we ascertain their order through Ultraviolet-Visible (UV-Vis) spectroscopy. The spectra for equimolar solutions in chloroform of the synthesized polysulfides along with that of the commercially available phenyl disulfide (PDS) as a reference were collected as shown in Figure $2 b$. The peak wavelength $(\lambda$, in $\mathrm{nm})$ increases as we move from PDS to PHS. ${ }^{31}$ The plot of peak wavelength as a function of square root of polysulfide order (n, i.e. number of sulfur atoms) shown in inset Figure 2b follows a linear trend as expected of phenyl polysulfide thus validating the order of the synthesized polysulfides. ${ }^{32}$

In addition, in order to confirm the purity of the synthesized polysulfides, electron impact mass spectrometry (EI-MS) was performed. The mass spectrum in Figure 2c has the parent ion peak at a m/z of 281.96 corresponding to the molar massfor PTS. Other daughter fragmentation peaks corresponding to that of $\mathrm{PhS}_{3} \mathrm{Ph}$ (phenyl trisulfide, $\mathrm{m} / \mathrm{z}=250$ ), $\mathrm{PhS}_{2} \mathrm{Ph}$ (phenyl disulfide, $\mathrm{m} / \mathrm{z}=218$ ), PhSS (phenyl perthiolate radical, $\mathrm{m} / \mathrm{z}=141$ ) and PhS $\cdot$ (phenyl thiolate radical, $\mathrm{m} / \mathrm{z}$ $=109$ ) are also evident. ${ }^{20}$ The absence of peaks beyond $\mathrm{m} / \mathrm{z}$ of 288 (supporting Figure S3) supports the clean formation of PTS. The mass spectrum in Figure 2d confirms the presence of PPS (m/z = 313.98) along with its fragments which include PTS. Trace amounts of PHS (m/z = 345.95) is also formed as evidenced by supporting Figure S4. The peak for a m/z of 345.92 in Figure 2e asserts the formation of PHS along with trace amounts of $\mathrm{PhS}_{7} \mathrm{Ph}$ (phenyl heptasulfide, $\mathrm{m} / \mathrm{z}=377.89$ ) indicated by supporting Figure S5. The spectroscopic data above substantiates the synthesis of the polysulfides as mainly governed by the equation in Figure 1a. 
The synthesized polysulfides were subject to electrochemical testing using CR-2032 coin cells. The cathode consisted of commercially available carbon nanotube (CNT) paper discs that function as the polysulfide reservoir, conductive substrate as well as the current collector as used in several studies. ${ }^{33-34}$ The cyclic voltammogram in Figure 3a shows three distinct reduction peaks in the cathodic scan which are present for all three polysulfides. The first peak at $2.4 \mathrm{~V}$ corresponds to the breakage of the central S-S bonds which increases with the polysulfide order owing to the lowering of their bond energy. ${ }^{35}$ The following reduction of the lithium polysulfides which occurs at $2.2 \mathrm{~V}$ is mediated by the formed phenyl persulfide and phenyl sulfide radicals. ${ }^{34}$, 36-38 This is followed by conversion of lithium polysulfides ( $\left.\mathrm{Li}_{2} \mathrm{~S}_{\mathrm{x}}, 2 \leq \mathrm{x} \leq 4\right)$ to lithium sulfide $\left(\mathrm{Li}_{2} \mathrm{~S}\right)$ and the formation of lithium thiophenolate $(\mathrm{PhSLi})$ at $2 \mathrm{~V} \cdot{ }^{39}$ The anodic scan illustrates that the charging reaction occurs in two steps. Majority of the conversion occurs at $2.3 \mathrm{~V}$ followed by the reversal to phenyl polysulfides at $2.43 \mathrm{~V}$.

Alongside this, the examination of the voltage profiles of the three polysulfides upon galvanostatic cycling at $\mathrm{C} / 2$ (Figure $3 \mathrm{~b}$ ) reveals the first discharge plateau occurs at $2.35 \mathrm{~V}$ for PHS and PPS whereas it is a sloping region until 2.25 V for PTS. This is followed by a sloping second plateau at 2.15 V for PTS and 2.2 V for PPS and PHS. The lower order lithium polysulfides formed at this stage $\left(\mathrm{Li}_{2} \mathrm{~S}_{\mathrm{x}}, 2 \leq \mathrm{x} \leq 4\right)$ transition to $\mathrm{Li}_{2} \mathrm{~S}$ at the $2 \mathrm{~V}$ plateau. We can see that, the higher voltage characteristic organopolysulfide transitions that occur above $2 \mathrm{~V}$ contribute to nearly $320 \mathrm{mAh} \mathrm{g}^{-1}$. This equates to $62 \%, 52 \%$, and $45 \%$ of the total capacity for PTS, PPS, and PHS, respectively, which is markedly different from the $25 \%$ obtained through the $2.4 \mathrm{~V}$ higher order polysulfide transition in Li-S batteries. ${ }^{40}$ The two electron-withdrawing phenyl rings attached to the sulfur chains elevate the electrochemical potential of sulfur; therefore it is beneficial for improving specific energy of lithium batteries. Additionally, the 
resonance in thiophenolate ion stabilizes it thus forming stable discharge products. This fundamentally changes the reaction mechanism compared to other organosulfur compounds. Following this, the physical and chemical transitions occurring at the cathode were probed using X-ray diffraction (XRD), X-ray photoelectron spectroscopy (XPS), and scanning electron microscopy (SEM). XRD pattern of the discharged cathodes in supporting Figure S6 exhibits the broadened (220) and (311) peaks for $\mathrm{Li}_{2} \mathrm{~S}$ and no characteristic peaks for PhSLi for all three polysulfides. This signals the formation of a near amorphous form of $\mathrm{Li}_{2} \mathrm{~S}$ and noncrystalline form of PhSLi on cell discharge, indicating a uniform mixing of $\mathrm{Li}_{2} \mathrm{~S}$ and $\mathrm{PhSLi}$ in the discharged electrode. On recharge, only the peaks for the bare CNT paper can be seen (supporting Figure S7) signaling the conversion of the solid discharge components to liquid phenyl polysulfides. To confirm the presence of PhSLi owing to its absence in XRD, we turned to XPS of the discharged cathode of PHS (Figure 3c). The presence of the doublet with S 2p $3 / 2$

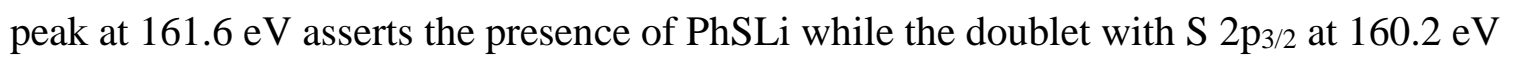
corroborates the $\mathrm{Li}_{2} \mathrm{~S}$ observed through XRD. Additionally, to capture the morphological transitions, SEM was performed. The micrograph of the discharged cathodes of PHS (Figure 3d), PPS (supporting Figure S8), and PTS (supporting Figure S9) all show the discharged products uniformly coated in what appears like a sheath over the CNT core. The lithium thiophenolate mediated discharge mechanism seems to assist in the spatially homogeneous distribution of discharge products. This uniform distribution of the discharge products in the CNT matrix as detected by the inset energy dispersive X-ray (EDX) ensures adequate electron transfer pathways and maximizes electrolyte penetration. It also enables excellent polysulfides confinement which results in high Coulombic efficiency (CE) and material utilization. ${ }^{41-42}$ The complete reversal to liquid polysulfides upon charge is revealed by the pristine nature of the CNT seen after washing 
the charged electrode (supporting Figure S10). In addition, the similar discharge voltage profiles observed in several cycles $\left(1^{\text {st }}, 250^{\text {th }}\right.$, and $\left.500^{\text {th }}\right)$ further confirm that the recharged product is electrochemically identical to the original phenyl polysulfide (supporting Figure S11).

Subsequently, these polysulfides were tested at 1C rate to capture the long-term cycling behavior of these materials (Figure 3e). PTS delivers $514 \mathrm{mAh} \mathrm{g}^{-1}$ which corresponds to 90\% material utilization. It is able to retain $65 \%$ of its initial capacity after 300 cycles. Meanwhile, PPS delivers $612 \mathrm{mAh} \mathrm{g}^{-1}$ which corresponds to $89.6 \%$ material utilization in the first cycle and also retains $75 \%$ this capacity post 300 cycles. The most stable of the polysulfides however, is PHS with an initial capacity of $650 \mathrm{mAh} \mathrm{g}^{-1}$ (84\% utilization) and a retention of $80 \%$ through 500 cycles. This increased cycling stability from PTS to PHS could be due to the increase in molecular size thus preventing its shuttle. Despite starting out with a low initial CE of $\sim 98 \%$, all polysulfides stabilize quickly and have an average CE of over 99.5\%. In addition to confinement of the phenyl polysulfides in the CNT pores as stated above, limiting the formation of long chain lithium polysulfides, and the sulfur concatenation mediated by the thiophenolate ion could all contribute to minimized shuttle effect and thus the high CE. Furthermore, excellent reversibility and sustenance of the same reaction pathway throughout the cycling of the phenyl polysulfides can be confirmed through the consistency observed in the voltage profile for PHS over 500 cycles (supporting Figure S11). This exemplary performance serves to cement the advantage of limiting the overall volume change at the cathode.

In addition to cycle life, high rate performance was also evaluated. PHS cathode was tested at various rates ranging from C/2 to $10 \mathrm{C}$ as in Figure 4a. PHS can deliver about $700 \mathrm{mAh}$ $\mathrm{g}^{-1}$ at low rates like $\mathrm{C} / 2$ and also manage to deliver $448 \mathrm{mAh} \mathrm{g}^{-1}$ and $409 \mathrm{mAh} \mathrm{g}^{-1}$ at high rates like 6C and 10C, respectively. This demonstrates a material utilization of over 50\% even at such 
high rates. The cell is able to cycle in $<12$ minutes at $6 \mathrm{C}$ and a little over 6 minutes at 10C. Moreover, the increase in overpotential, i.e., $0.35 \mathrm{~V}$ from C/2 to 10C is modest (supporting Figure S12), showing the excellent power performance of this material. To put this in a practical perspective we turn to Ragone plots wherein the interplay between energy and power is appropriately highlighted. The gravimetric Ragone plot in supporting Figure S13 shows that, at a material level, over $1500 \mathrm{Wh} \mathrm{kg}^{-1}$ can be obtained under low demands such as $800 \mathrm{~W} \mathrm{~kg}^{-1}$. However, even under pressing demand scenarios $\left(13 \mathrm{~kW} \mathrm{~kg}^{-1}\right)$, a high specific energy of $725 \mathrm{Wh}$ $\mathrm{kg}^{-1}$ can be obtained. Even on a volumetric basis (supporting Figure S14) $800 \mathrm{Wh} \mathrm{L}^{-1}$ can be achieved at a power demand corresponding to $13 \mathrm{~kW} \mathrm{~L}^{-1}$.

Many recent works have rightly shown that low electrolyte to active material ratios are a critical aspect of materials design for true high energy density sulfur-based batteries. ${ }^{7-10}$ Keeping in mind that a high loading cathode with minimal electrolyte is essential for achieving high cell level specific energy, we tested a high loading (9 $\mu \mathrm{L}$ or $11.9 \mathrm{mg}$ ) of PHS which comprised $68 \%$ of the cathode's mass with a $3 \mu \mathrm{L} \mathrm{mg}^{-1}$ electrolyte ratio at C/10 rate. As seen in Figure 3d, this cathode can yield $7.6 \mathrm{mAh} \mathrm{cm}^{-2}$ of areal capacity which is much higher than that of commercial Li-ion cells. ${ }^{43}$ Stable cycling is achieved for 50 cycles with $82 \%$ capacity retention. The observed fading is attributed to aggressive electrolyte consumption and failure of lithium anode (supporting Figure S15). At an active material level, PHS can offer an impressive specific energy of $1,302 \mathrm{Wh} \mathrm{kg}^{-1}$ and an energy density of $1,720 \mathrm{Wh} \mathrm{L}^{-1}$ based on the first cycle. To highlight the improved performance of PHS over sulfur cathodes when operating under low-electrolyte, i.e., lean conditions, PHS cells were compared to solid lithium polysulfide-based cells with the same substrate and theoretical cathode capacity were tested at three electrolyte-to-sulfur ratios namely 3, 5, and $10 \mu \mathrm{Lg}^{-1}$. Inspection of the first cycle voltage profile (supporting Figure S16) of $3 \mu \mathrm{L}$ 
$\mathrm{mg}^{-1}$ based cell shows failure to operate normally while the cell with $5 \mu \mathrm{L} \mathrm{mg}{ }^{-1}$ exhibits high overpotentials. Only at $10 \mu \mathrm{L} \mathrm{mg}^{-1}$ did sulfur cells achieve first cycle performance comparable to the PHS, but penalties to overpotential and capacity remained. Assessment of cycling behavior (supporting Figure S17) of these cells clearly shows failure at $3 \mu \mathrm{L} \mathrm{mg}^{-1}$ and rapid degradation at $5 \mu \mathrm{L} \mathrm{mg}{ }^{-1}$. Even at $10 \mu \mathrm{L} \mathrm{mg}^{-1}$ only $69 \%$ of sulfur can be utilized. For reference, $80 \%$ of PHS can be utilized even at $3 \mu \mathrm{L} \mathrm{mg}^{-1}$ cycling conditions. Moreover, the CE of the operating sulfur cells (supporting Figure S18) is 89\% compared to that of PHS at 99\%. This superior cycling performance of PHS coupled with a reduced inactive electrolyte fraction translates to nearly 100 Wh $\mathrm{kg}^{-1}$ higher specific energy than the sulfur system at a cell level as conveyed in supporting Figure S19. While this work only serves to demonstrate the potential of this class of materials, further engineering and optimization could certainly enable much higher specific energies from these batteries.

\section{Conclusion}

In summary, we demonstrate that high purity phenyl polysulfides can be synthesized in a facile, low cost, one-pot route that is scalable. These polysulfides are high-sulfur-content compounds with high theoretical capacities in the order of PHS > PPS > PTS and high energy densities. They exhibit long high discharge voltage plateaus due to the electron-withdrawing phenyl rings attached to the sulfur chains and each polysulfide has its own characteristic voltage profile. In Li half cells, they show excellent cyclability up to 500 cycles due to reduced volume expansion in the cathode along with superior rate performance. Importantly, high-order PHS shows respectable performance under lean electrolyte operation, which outperforms lithium polysulfide cathode in terms of material utilization, Coulombic efficiency, and specific energy. 
These multifaceted advantages provide the motivation for further exploration of this class of materials towards a truly high energy density sulfur-based battery system.

\section{Supporting Information}

Supporting Information Available: experimental details, volume change calculations along with additional materials characterization and electrochemical data. This material is available free of charge via the Internet at http://pubs.acs.org

\section{Acknowledgements}

YF acknowledges the Release Time for Research grant from Office of the Vice Chancellor for Research at Indiana University-Purdue University Indianapolis and the support from Thousand Youth Talents Program of China. We would like to acknowledge the Integrated Nanosystems Development Institute (INDI) for use of their Bruker D8 Discover XRD Instrument, which was awarded through the NSF grant MRI-1429241 and for use of their JEOL7800F Field Emission SEM, which was awarded through NSF grant MRI-1229514. We thank the Department of Chemistry and Chemical Biology at IUPUI for the use of their FTIR facility. We also thank Dr. Yaroslav Losovyj at the Nanoscale Characterization Facility at Indiana University for XPS measurements.

\section{Author contributions}

A.B. proposed the concept of the battery and did the main measurements and analysis under the Y.F.'s supervision. M.E.B. assisted in acquiring FTIR data. Y.C. performed the SEM. J.K. collected and assisted in the analysis of EI-MS data. A.B. and Y.F. prepared the manuscript. All the authors discussed the results and commented on the manuscript. 


\section{Competing interests}

The authors declare no competing financial interests.

\section{Corresponding Author}

yfu@zzu.edu.cn(Y.Fu)

\section{References}

1. Goodenough, J. B.; Park, K.-S., The Li-ion rechargeable battery: a perspective. J. Am. Chem. Soc. 2013, 135, 1167-1176.

2. Armand, M.; Tarascon, J. M., Building better batteries. Nature 2008, 451, 652-657.

3. Manthiram, A., Materials Challenges and Opportunities of Lithium Ion Batteries. J. Phys. Chem. Lett. 2011, 2, 176-184.

4. Goodenough, J. B.; Kim, Y., Challenges for Rechargeable Li Batteries. Chem. Mater. 2010, 22, 587-603.

5. Manthiram, A.; Fu, Y.; Su, Y.-S., Challenges and prospects of lithium-sulfur batteries. Acc. Chem. Res. 2012, 46, 1125-1134.

6. Manthiram, A.; Fu, Y.; Chung, S.-H.; Zu, C.; Su, Y.-S., Rechargeable lithium-sulfur batteries. Chem. Rev. 2014, 114, 11751-11787.

7. Hagen, M.; Hanselmann, D.; Ahlbrecht, K.; Maça, R.; Gerber, D.; Tübke, J., LithiumSulfur Cells: The Gap between the State-of-the-Art and the Requirements for High Energy Battery Cells. Adv. Energy Mater. 2015, 5, 1401986.

8. McCloskey, B. D., Attainable Gravimetric and Volumetric Energy Density of Li-S and Li Ion Battery Cells with Solid Separator-Protected Li Metal Anodes. J. Phys. Chem. Lett. 2015, 6, 4581-4588.

9. Fu, C.; Guo, J., Challenges and current development of sulfur cathode in lithium-sulfur battery. Curr. Opin. Chem. Eng. 2016, 13, 53-62.

10. Peng, H. J.; Huang, J. Q.; Cheng, X. B.; Zhang, Q., Review on High-Loading and HighEnergy Lithium-Sulfur Batteries. Adv. Energy Mater. 2017, 7, 1700260.

11. Guo, W.; Fu, Y., A Perspective on Energy Densities of Rechargeable Li-S Batteries and Alternative Sulfur-Based Cathode Materials. Energy Environ. Mater. 2018, 1, 20-27.

12. Wang, H.; Yang, Y.; Liang, Y.; Robinson, J. T.; Li, Y.; Jackson, A.; Cui, Y.; Dai, H., Graphene-Wrapped Sulfur Particles as a Rechargeable Lithium-Sulfur Battery Cathode Material with High Capacity and Cycling Stability. Nano Lett. 2011, 11, 2644-2647.

13. Evers, S.; Nazar, L. F., Graphene-enveloped sulfur in a one pot reaction: a cathode with good coulombic efficiency and high practical sulfur content. Chem. Commun. 2012, 48, 1233-1235.

14. Seh, Z. W.; Li, W.; Cha, J. J.; Zheng, G.; Yang, Y.; McDowell, M. T.; Hsu, P.-C.; Cui, Y., Sulphur- $\mathrm{TiO}_{2}$ yolk-shell nanoarchitecture with internal void space for long-cycle lithium-sulphur batteries. Nat. Commun. 2013, 4, 1331. 
15. Fu, Y.; Manthiram, A., Core-shell structured sulfur-polypyrrole composite cathodes for lithium-sulfur batteries. RSC Adv. 2012, 2, 5927-5929.

16. Wu, F.; Chen, J.; Chen, R.; Wu, S.; Li, L.; Chen, S.; Zhao, T., Sulfur/Polythiophene with a Core/Shell Structure: Synthesis and Electrochemical Properties of the Cathode for Rechargeable Lithium Batteries. J. Phys. Chem. C 2011, 115, 6057-6063.

17. Yang, Y.; Yu, G.; Cha, J. J.; Wu, H.; Vosgueritchian, M.; Yao, Y.; Bao, Z.; Cui, Y., Improving the Performance of Lithium-Sulfur Batteries by Conductive Polymer Coating. ACS Nano 2011, 5, 9187-9193.

18. Barai, P.; Mistry, A.; Mukherjee, P. P., Poromechanical effect in the lithium-sulfur battery cathode. Extreme Mechanics Letters 2016, 9, 359-370.

19. Waluś, S.; Offer, G.; Hunt, I.; Patel, Y.; Stockley, T.; Williams, J.; Purkayastha, R., Volumetric expansion of Lithium-Sulfur cell during operation - Fundamental insight into applicable characteristics. Energy Storage Materials 2018, 10, 233-245.

20. Cerda, M. M.; Hammers, M. D.; Earp, M. S.; Zakharov, L. N.; Pluth, M. D., Applications of Synthetic Organic Tetrasulfides as $\mathrm{H}_{2} \mathrm{~S}$ Donors. Org. Lett. 2017, 19, 2314-2317.

21. Derbesy, G.; Harpp, D. N., A simple method to prepare unsymmetrical di- tri- and tetrasulfides. Tetrahedron Lett. 1994, 35, 5381-5384.

22. Chakravarti, G. C., CX.-Action of sulphur monochloride on mercaptans. J. Chem. Soc., Trans. 1923, 123, 964-968.

23. Kawase, A.; Shirai, S.; Yamoto, Y.; Arakawa, R.; Takata, T., Electrochemical reactions of lithium-sulfur batteries: an analytical study using the organic conversion technique. Phys. Chem. Chem. Phys. 2014, 16, 9344-9350.

24. Nobuo, Y.; Mutsuhisa, F.; Masayuki, N.; Toshikazu, T., Direct Preparation of Anhydrous Sodium Oligosulfides from Metal Sodium and Elemental Sulfur in Aprotic Organic Media Directed toward Synthesis of Silane Coupling Agent. Chem. Lett. 2002, 31, 454455.

25. Vineyard, B. D., Versatility and the mechanism of the n-butyl-amine-catalyzed reaction of thiols with sulfur. J. Org. Chem. 1967, 32, 3833-3836.

26. Steudel, R., The Chemistry of Organic Polysulfanes R-Sn-R ( $>$ 2). Chem. Rev. 2002, 102, 3905-3946.

27. Myung, S.-T.; Maglia, F.; Park, K.-J.; Yoon, C. S.; Lamp, P.; Kim, S.-J.; Sun, Y.-K., Nickel-Rich Layered Cathode Materials for Automotive Lithium-Ion Batteries:

Achievements and Perspectives. ACS Energy Lett. 2017, 2, 196-223.

28. Das, P.; Ray, S.; Bhaumik, A.; Banerjee, B.; Mukhopadhyay, C., Cubic $\mathrm{Ag}_{2} \mathrm{O}$ nanoparticle incorporated mesoporous silica with large bottle-neck like mesopores for the aerobic oxidative synthesis of disulfide. RSC Adv. 2015, 5, 6323-6331.

29. Minoura, Y.; Moriyoshi, T., Infra-red spectra of dibenzyl polysulphides. Trans. Faraday Soc. 1963, 59, 1504-1509.

30. Tsurugi, J.; Nakabayashi, T., Organic Polysulfides. II.1 Polymorphism in Dibenzhydryl Tetrasulfide. J. Org. Chem. 1960, 25, 1744-1747.

31. Tsurugi, J.; Nakabayashi, T., Synthesis of Dibenzhydryl and Dibenzyl Penta- and Hexasulfides. J. Org. Chem. 1959, 24, 807-810.

32. Minoura, Y.; Moriyoshi, T., Ultra-violet absorption spectra and molecular refractions of dibenzyl mono-, di-, tri-, tetra-, penta-, hexa-, hepta-, octa-sulphides. Trans. Faraday Soc. 1963, 59, 1019-1025. 
33. Fu, Y.; Su, Y.-S.; Manthiram, A., Highly Reversible Lithium/Dissolved Polysulfide Batteries with Carbon Nanotube Electrodes. Angew. Chem. Int. Ed. 2013, 52, 6930-6935.

34. Wu, M.; Cui, Y.; Bhargav, A.; Losovyj, Y.; Siegel, A.; Agarwal, M.; Ma, Y.; Fu, Y., Organotrisulfide: A High Capacity Cathode Material for Rechargeable Lithium Batteries. Angew. Chem. Int. Ed. 2016, 55, 10027-10031.

35. Denk, M. K., The Variable Strength of the Sulfur-Sulfur Bond: 78 to 41 kcal - G3, CBSQ, and DFT Bond Energies of Sulfur $\left(\mathrm{S}_{8}\right)$ and Disulfanes XSSX (X = H, F, Cl, $\mathrm{CH}_{3}, \mathrm{CN}$, $\mathrm{NH}_{2}$, OH, SH). Eur. J. Inorg. Chem. 2009, 2009, 1358-1368.

36. Chen, S.; Dai, F.; Gordin, M. L.; Yu, Z.; Gao, Y.; Song, J.; Wang, D., Functional Organosulfide Electrolyte Promotes an Alternate Reaction Pathway to Achieve High Performance in Lithium-Sulfur Batteries. Angew. Chem. Int. Ed. 2016, 55, 4231-4235.

37. Bhargav, A.; Patil, S. V.; Fu, Y., A phenyl disulfide@CNT composite cathode for rechargeable lithium batteries. Sustainable Energy Fuels 2017, 1, 1007-1012.

38. Shuru, C.; Daiwei, W.; Yuming, Z.; Donghai, W., Superior Performance of a LithiumSulfur Battery Enabled by a Dimethyl Trisulfide Containing Electrolyte. Small Methods 2018, 2, 1800038.

39. Guo, W.; Wawrzyniakowski, Z.; Cerda, M.; Bhargav, A.; Pluth, M.; Ma, Y.; Fu, Y., Bis(aryl) Tetrasulfides as Cathode Materials for Rechargeable Lithium Batteries. Chem. Eur. J. 2017, 23, 16941-16947.

40. Su, Y.-S.; Fu, Y.; Cochell, T.; Manthiram, A., A strategic approach to recharging lithiumsulphur batteries for long cycle life. Nat. Commun. 2013, 4, 2985.

41. Hua, W.; Yang, Z.; Nie, H.; Li, Z.; Yang, J.; Guo, Z.; Ruan, C.; Chen, X. a.; Huang, S., Polysulfide-Scission Reagents for the Suppression of the Shuttle Effect in Lithium-Sulfur Batteries. ACS Nano 2017, 11, 2209-2218.

42. $\quad$ Preefer, M. B.; Oschmann, B.; Hawker, C. J.; Seshadri, R.; Wudl, F., High Sulfur Content Material with Stable Cycling in Lithium-Sulfur Batteries. Angew. Chem. Int. Ed. 2017, 56, 15118-15122.

43. Gallagher, K. G.; Trask, S. E.; Bauer, C.; Woehrle, T.; Lux, S. F.; Tschech, M.; Lamp, P.; Polzin, B. J.; Ha, S.; Long, B.; Wu, Q.; Lu, W.; Dees, D. W.; Jansen, A. N., Optimizing Areal Capacities through Understanding the Limitations of Lithium-Ion Electrodes. $J$. Electrochem. Soc. 2016, 163, A138-A149. 
(a)

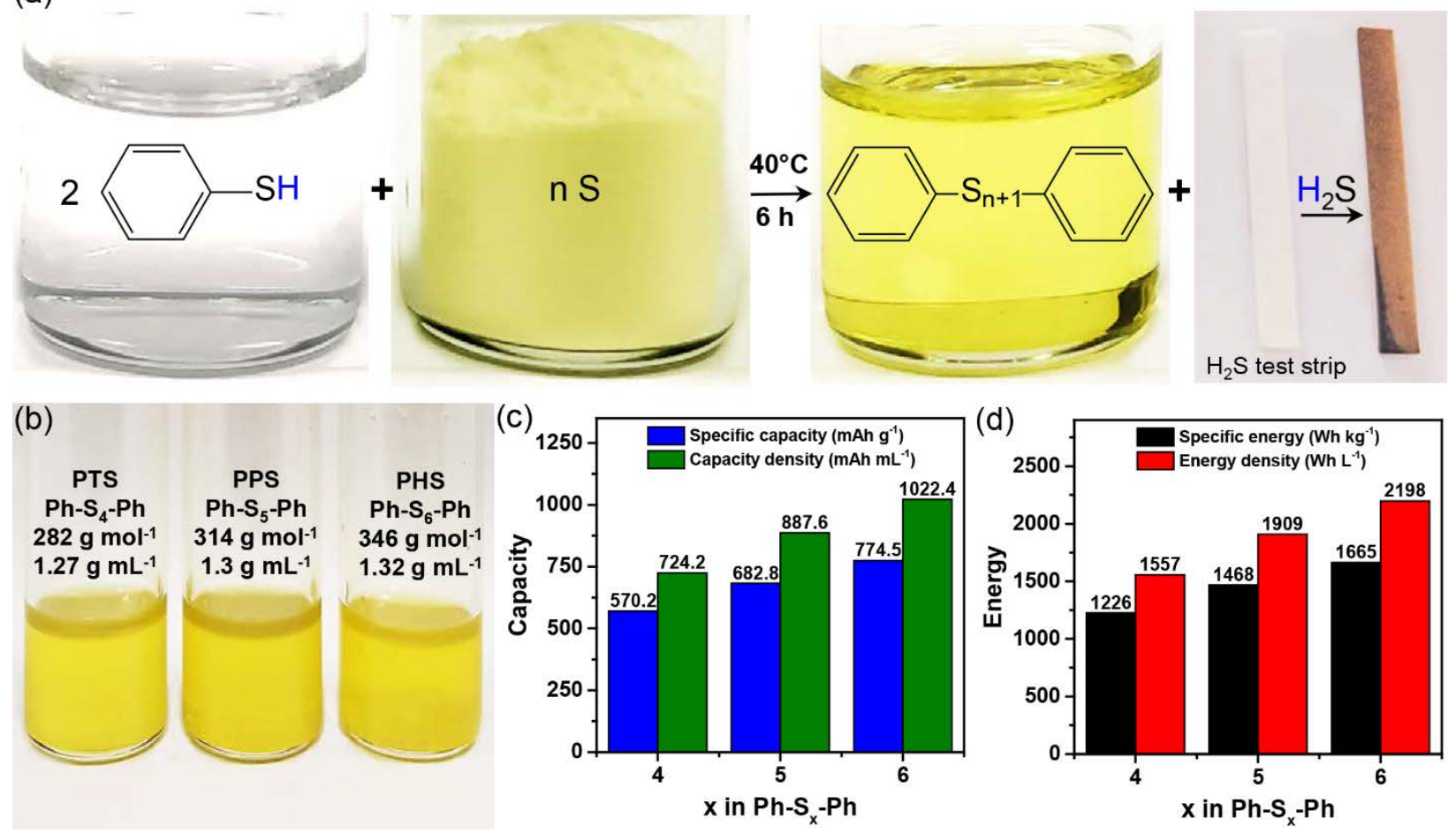

Figure 1. (a) Equation along a visual representation of the phenyl polysulfide synthesis process.

Lead acetate based test strip was used to confirm the $\mathrm{H}_{2} \mathrm{~S}$ evolution. (b) Optical image of the liquid polysulfides along with their properties. (c) Theoretical specific capacities (mAh g${ }^{-1}$ ) and capacity densities ( $\mathrm{mAh} \mathrm{mL}^{-1}$ ) of the different polysulfides and their (d) theoretical specific energies $\left(\mathrm{Wh} \mathrm{kg}^{-1}\right)$ and energy densities $\left(\mathrm{Wh} \mathrm{L}^{-1}\right)$. 


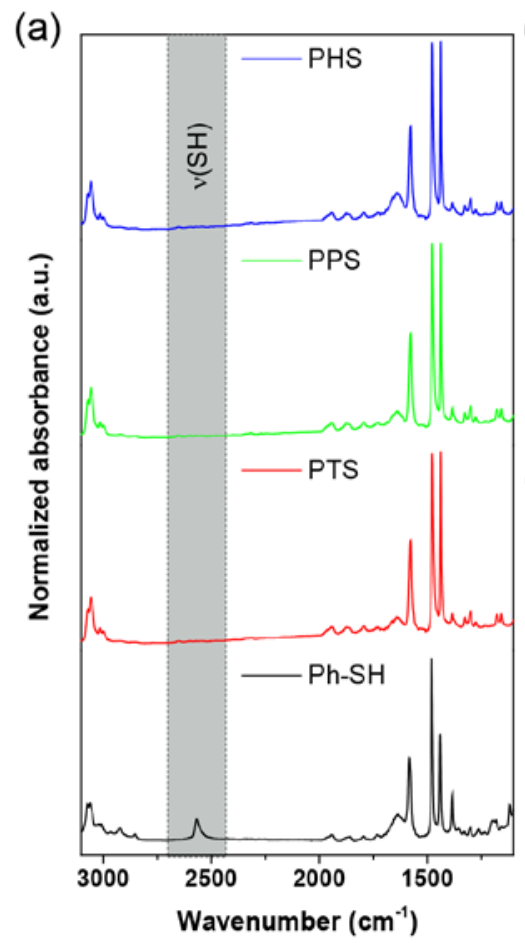

(b)

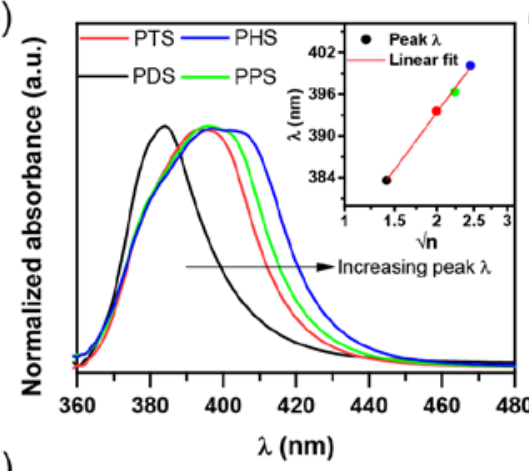

(d)

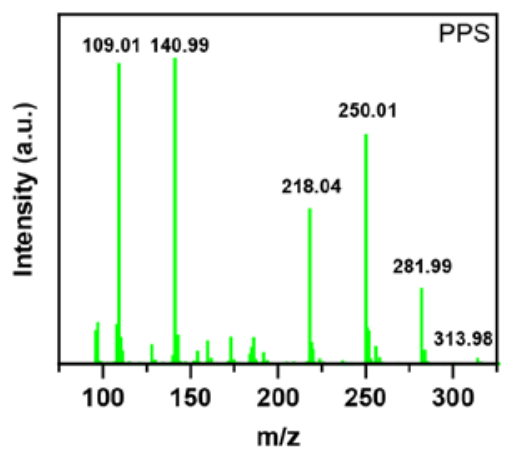

(c)

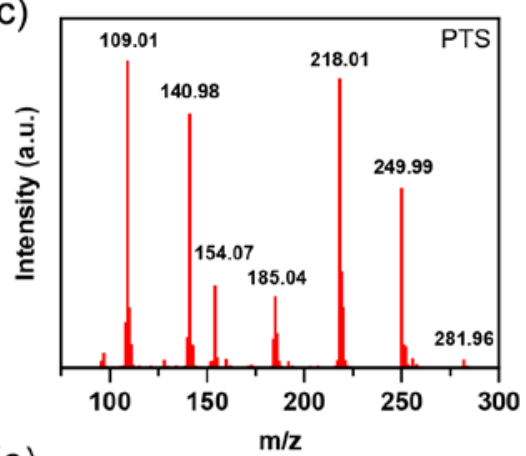

(e)

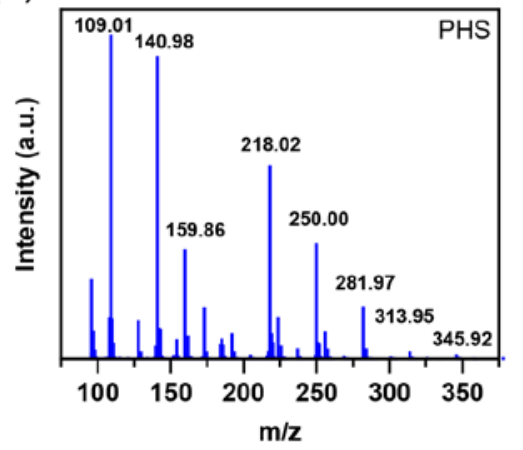

Figure 2. (a) FTIR spectrum of benzenethiol (Ph-SH) and the synthesized phenyl polysulfides.

(b) UV-Vis spectra of equimolar solutions of different phenyl polysulfides along with that of phenyl disulfide (PDS) as reference. Inset of (b) shows the linear increase in peak $\lambda$ as a function of number of sulfur atoms. EI-MS spectrum of (c) phenyl tetrasulfide (PTS), (d) phenyl pentasulfide (PTS), and (e) phenyl hexasulfide (PHS). 


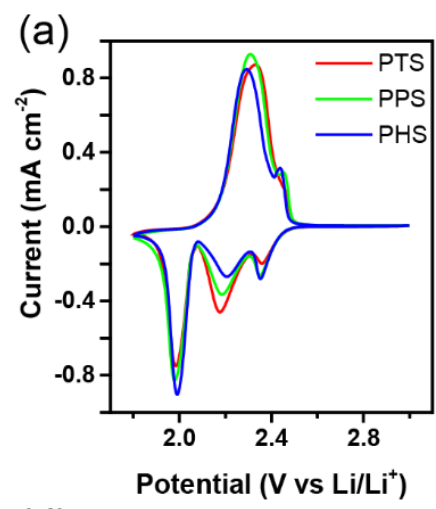

(d)

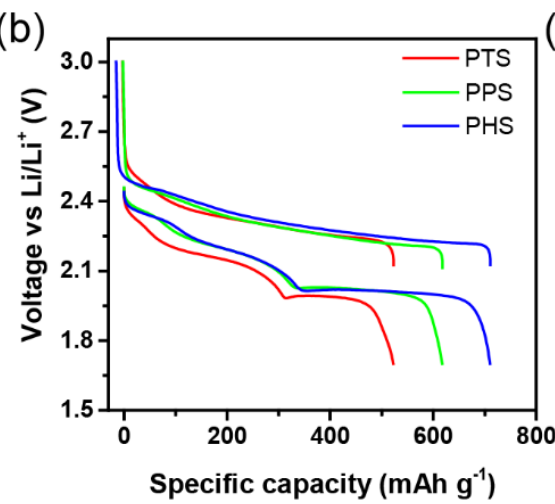

(e)
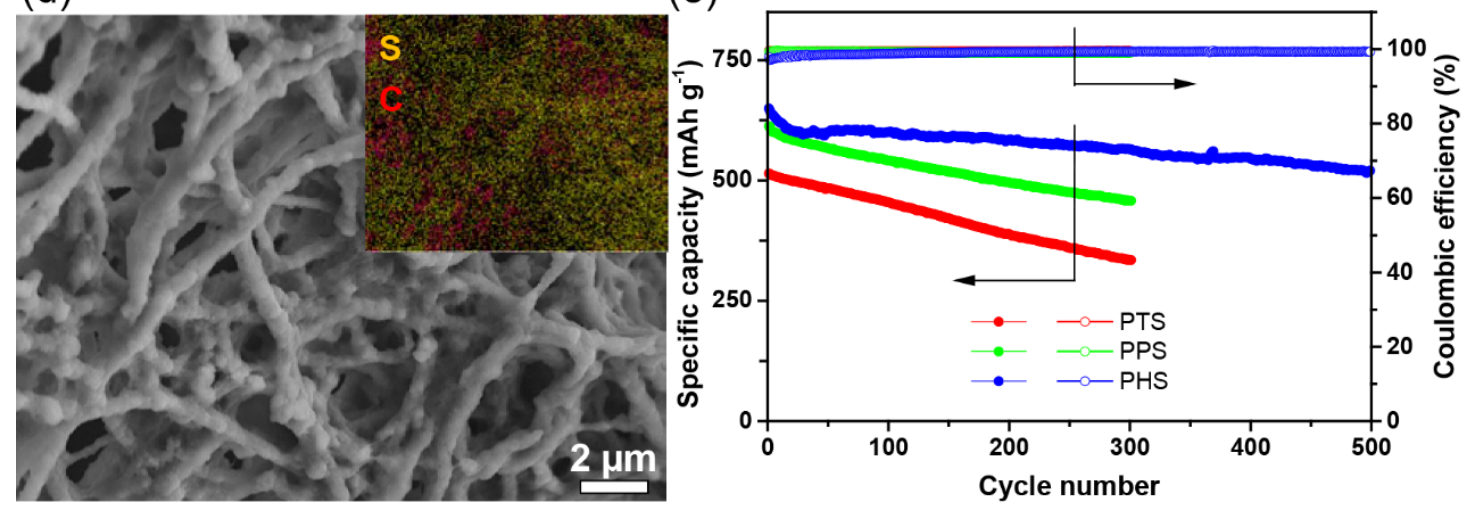

Figure 3. (a) Cyclic voltammetry (CV) of the phenyl polysulfide cathodes performed at $0.05 \mathrm{mV}$ $\mathrm{s}^{-1}$. (b) Voltage profile of the polysulfide cathodes cycled at C/2. (c) Sulfur 2p XPS spectrum of the PHS cathode in the discharged state. (d) SEM of the PHS cathode in the discharged state along with its inset EDX mapping and (e) long term cycling performance of the polysulfides at 1C. The cycling rate was based on active material mass in the cathode with $1 \mathrm{C}_{\mathrm{PTS}}=570 \mathrm{~mA} \mathrm{~g}^{-1}$, $1 \mathrm{C}_{\mathrm{PPS}}=683 \mathrm{~mA} \mathrm{~g}^{-1}$, and $1 \mathrm{C}_{\mathrm{PHS}}=775 \mathrm{~mA} \mathrm{~g}^{-1}$. 
(a)

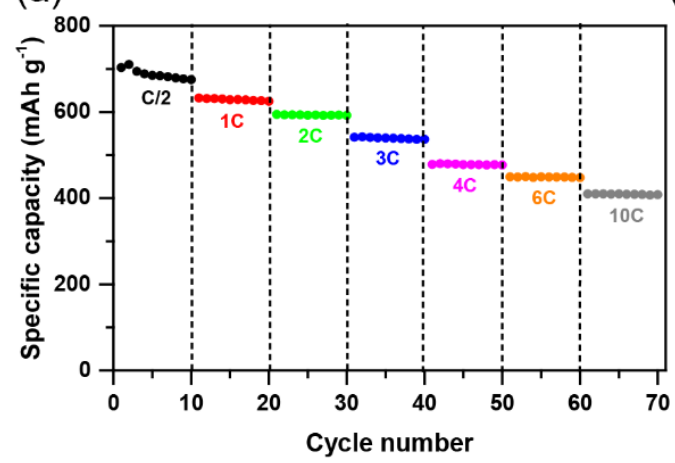

(b)

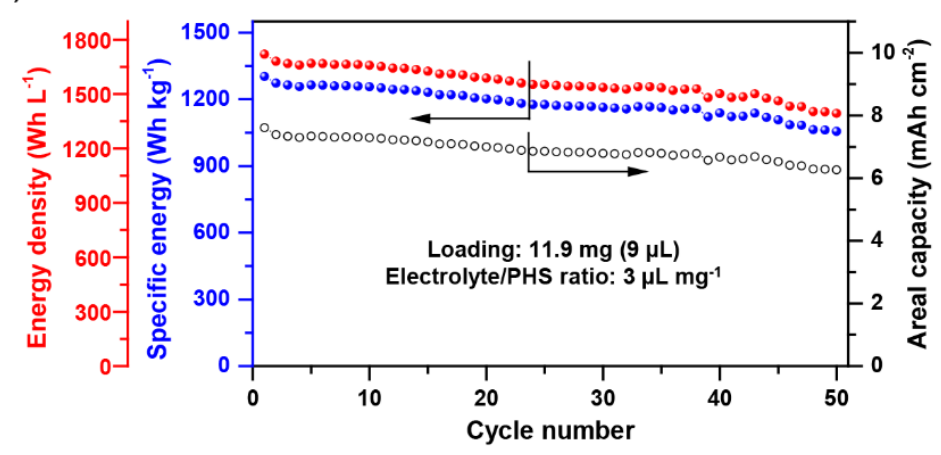

Figure 4. (a) Rate performance of PHS cathode and (b) performance of high loading, low electrolyte/PHS ratio cell cycled at C/10. The cycling rate was based on active material mass in the cathode with $1 \mathrm{C}_{\mathrm{PTS}}=570 \mathrm{~mA} \mathrm{~g}^{-1}, 1 \mathrm{C}_{\mathrm{PPS}}=683 \mathrm{~mA} \mathrm{~g}^{-1}$, and $1 \mathrm{C}_{\mathrm{PHS}}=775 \mathrm{~mA} \mathrm{~g}^{-1}$. 


\section{Table of Contents Figure}

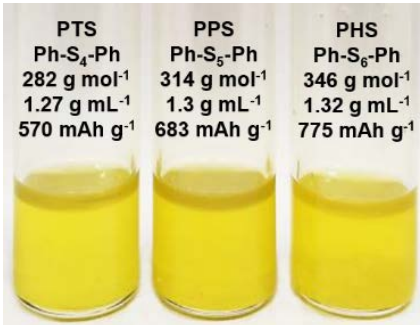

